\title{
Prototipação de Chatterbots como Método de Aprendizagem em Cursos de Computação: uma experiência em sala de aula
}

\author{
Sílvia M. W. Moraes ${ }^{1}$, André L. L. Santos' ${ }^{1}$ Matheus Redecker ${ }^{1}$ \\ ${ }^{1}$ Faculdade de Informática - Pontifícia Universidade Católica do Rio Grande do Sul \\ (PUCRS) \\ Caixa Postal 1429 - 90619-900 - Porto Alegre - RS - Brazil \\ silvia.moraes@pucrs.br, andre.leonhardt.santos@gmail.com, \\ matheus.redecker@acad.pucrs.br
}

\begin{abstract}
This paper describes a classroom experiment in which we used prototyping of chatbots as a learning method. We conducted this experiment for Artificial Intelligence students from the undergraduate course of Information Systems. In this paper, we detail the steps of method used and the results obtained. In our study, we perceived greater engagement of students in the proposed activities and their learning gains.
\end{abstract}

Resumo. Este artigo relata uma experiência em sala de aula na qual a prototipação de chatbots foi utilizada como método de aprendizagem. Essa experiência foi realizada em uma turma de Inteligência Artificial do curso de Sistemas de Informação. Neste artigo, são detalhadas as etapas do método usado, bem como os resultados obtidos. Nós percebemos um maior engajamento dos alunos nas atividades propostas e ganhos em suas aprendizagens.

\section{Introdução}

A motivação é fundamental em qualquer processo de aprendizagem. Ela influencia diretamente no tempo dedicado, no esforço empregado, na persistência e na forma como os estudantes enfrentam os obstáculos que aparecem ao longo desse processo (Bakar, 2014). Diferentes abordagens têm surgido como formas de estimular e aumentar o engajamento dos alunos nas atividades que promovem a aprendizagem. Dentre essas abordagens, o uso de agentes conversacionais é comum.

$\mathrm{Na}$ área educacional, especialmente em e-learning, o interesse em agentes conversacionais é grande e tem como justificativa as características desses agentes. Eles provêem uma interface mais natural e prática ao aluno. São capazes de oferecer um suporte pessoal, de reconhecer pontos fortes, interesses e habilidades individuais dos alunos. Além disso, podem acelerar o processo de aprendizagem ao atuarem como instigadores dos tópicos em estudo, resultando em um maior engajamento e na independência dos estudantes (Kerly et al., 2009; Griol et al., 2011; Moreno et al., 2015; Ramayana et al., 2014).

Conceitualmente, os agentes conversacionais são sistemas de diálogo que têm como objetivo a comunicação, em linguagem natural, com usuários a fim de auxiliá-los de alguma forma (Jurafsky e Martin, 2005; Al-Zubaide e Issa, 2011). Esses agentes são conhecidos como os chatterbots (ou chatbots) e respondem a perguntas de tal forma que os usuários têm a impressão de estarem conversando com uma pessoa e não com um programa de computador. 
A maioria das aplicações de chatbots, na área educacional, giram em torno de sistemas de tutoria (Deters et al., 2006), de perguntas e respostas (Amorim et al., 2011), de conversação destinada à aprendizagem de uma nova língua (Ferreira e Uchuoa, 2006) e, também, em sistemas de diálogos cujo fím é estimular a reflexão e as habilidades metacognitivas dos alunos (Tamayo e Pérez-Marín, 2012). É usual atuarem, nessas aplicações, como agentes pedagógicos e parceiros no processo de aprendizagem (Kerly et al., 2009).

Há ainda algumas abordagens, como as que apresentamos neste artigo, que utilizam a prototipação do chatbot como método motivador e facilitador da aprendizagem (Shaw, 2012; Bii, 2013; Benotti et al., 2014). Em tais abordagens, o objetivo é instigar a busca pelo conhecimento usando como estímulo a construção de uma base de dados de um agente conversacional. Várias pesquisas nesse sentido têm conseguido bons resultados, principalmente no que se refere ao aumento da motivação e, consequentemente, da participação dos alunos, especialmente quando a área de formação dos alunos está relacionada à Ciência da Computação. Benotti et al. (2014) afirmam que há vários clubes de programação propondo a prototipação de chatbots como um método para aumentar o interesse dos estudantes em Ciência da Computação.

Neste artigo, relatamos uma experiência em sala de aula na qual usamos a prototipação de chatbots como uma forma de aumentar a motivação e o engajamento dos alunos na aprendizagem de conceitos de Inteligência Artificial (IA). As bases dos chatbots foram construídas em Artificial Intelligence Markup Language (AIML ${ }^{1}$ ). As experiências foram realizadas em uma turma do curso de Sistemas de Informação (SI), durante o ano de 2015. Os resultados foram animadores e obtivemos ganhos na aprendizagem dos alunos.

Este artigo está organizado em 5 Seções. Na Seção 2 são comentados alguns trabalhos relacionados a este. Na Seção 3 é descrito o método motivacional baseado na prototipação de chatbots. Na Seção 4 é detalhada a experiência de prototipação realizada com os alunos e os resultados obtidos. E, por fim, na Seção 5 são apresentadas as considerações finais e os trabalhos futuros.

\section{Trabalhos Relacionados}

O uso de chatbots com propósito educacional não é novidade, no entanto a prototipação deles como um método de ensino e de aprendizagem é mais recente. Bii (2013) usa a tecnologia de chatbots para ensinar Biologia. Ele propôs aos alunos a construção de uma base AIML sobre Genética. Seu estudo mostrou que a programação do chatbot pelos alunos, além de estimular a colaboração, cooperação e interação, tornou a aprendizagem mais ativa, construtiva, criativa e social.

Benotti et al. (2014) não só tentam tornar o estudo mais atrativo, usando como método de ensino a prototipação de chatbots, como também tentam medir a efetividade desse método. Os autores aplicam o método usando como estratégia pedagógica a gamificação. O objetivo dos autores é despertar o interesse dos alunos pela área de Ciência da Computação. Eles analisam o engajamento dos alunos por meio de indicadores como participação, concentração, entusiasmo e interesse na realização das tarefas. Os resultados obtidos por esses pesquisadores foram animadores. Shaw (2012) igualmente obteve bons resultados usando a programação de chatbots para ensinar conceitos básicos em cursos introdutórios de Computação.

1 Mais detalhes sobre AIML são apresentados na seção 4. 
$\mathrm{Na}$ área de administração, Domingues e Rothermel (2008) relatam a experiência de ensinar métodos e técnicas de pesquisa por meio da prototipação de chatbots. Segundo as autoras, depois que os alunos dominaram a tecnologia, eles formularam em média mais de 10 templates de perguntas em apenas 15 minutos de prototipação. As autoras também relatam que os alunos afirmaram que a prototipação auxiliou na compreensão dos conceitos da disciplina.

O método e a experiência que descrevemos neste artigo diferem na forma, mas no propósito são semelhantes aos trabalhos aqui descritos. A seção seguinte detalha o método que definimos.

\section{Método de Aprendizagem baseado em Prototipação de Chatbots}

Este método foi concebido para alunos do curso de Sistemas de Informação com o objetivo de aumentar o interesse e, consequentemente, a aprendizagem na disciplina de Inteligência Artificial. A meta era a construção de um chatbot, sendo que a sua base de conhecimento deveria ser desenvolvida em conjunto pelos alunos. O método prevê 3 etapas:

- Etapa 1 - Familiarizando-se com chatbots: Antes de prototipar um chatbot é importante que o aluno conheça um. Nessa etapa, os alunos são convidados a avaliar um chatbot disponível na web. Conduzimos essa etapa fornecendo aos alunos questionários de pré e pós-teste.

- Etapa 2 - Construindo um chatbot: Conhecendo um chatbot é possível discutir formas de implementação, ambientes, linguagens, dificuldades e desafios inerentes à sua construção. A prototipação faz o aluno pensar sobre o conhecimento que o agente precisa dispor e aprende construindo. Nessa etapa, a turma deve ser organizada em equipes. Nas equipes, cada estudante deve ter um papel. Os papéis são aqueles usualmente utilizados para designar atribuições em projetos de desenvolvimento de software, tais como: analista, arquiteto, testador, desenvolvedor e gerente de projeto. Nessa etapa, o chatbot é projetado e construído pelos alunos.

- Etapa 3 - Avaliando um chatbot. Nessa etapa, as equipes devem conduzir a avaliação do chatbot construído pela turma. Cada equipe deve avaliar o chatbot construído junto a outros usuários. Essa etapa estimula o pensamento crítico e a reflexão sobre as dificuldades inerentes à avaliação de agentes, bem como provoca uma reflexão quanto a recursos de IA que poderiam ser inseridos para deixar o chatbot mais inteligente e com uma interação mais natural e amigável.

O método proposto foi aplicado em uma disciplina de IA para o curso de SI, no entanto entendemos que ele pode ser adaptado para outras disciplinas em diferentes cursos da área de Computação. Na próxima seção, relatamos a experiência de aplicação do método em sala de aula.

\section{Relato da Experiência em Sala de Aula}

A primeira experiência foi aplicada em uma turma da disciplina de IA com 33 alunos, durante o semestre de 2015/01. Todos os alunos da turma cursavam SI. A prototipação do chatbot foi, então, proposta como atividade à turma. Seu objetivo era instigar o interesse em agentes reativos, que era o tópico em estudo. No contexto da disciplina de 
IA, o método é bem pertinente. Agentes conversacionais baseados apenas em AIML são reativos. Desta forma, os alunos, além de relacionarem a teoria à prática, por meio da implementação de um agente reativo, também teriam que se aprofundar nos tópicos da disciplina para construir as perguntas e respostas da base desse agente.

\subsection{Familiarizando-se com Chatbot}

Nesta etapa, o objetivo era provocar a interação dos alunos com chatbots conhecidos a fim de familiarizá-los com agentes conversacionais e suas aplicações. Para isso, após uma breve exposição conceitual sobre chatbots, propomos uma atividade individual que consistia em interagir por alguns minutos com o chatbot $\mathrm{ED}^{2}$. Por meio da interação, os alunos tinham que descobrir aspectos relacionados à personalidade e ao propósito, assim como as características e limitações, do ponto de vista computacional, desse agente. Cabe mencionar que escolhemos este chatbot por estar em Língua Portuguesa, ser bem construído, e estar disponível na web. A atividade proposta envolvia o preenchimento de questionários. Usamos um questionário de pré-teste (Figura 1) para medirmos o conhecimento prévio do aluno sobre agentes conversacionais e um de pós-teste (Figura 2) para que ele descrevesse e avaliasse sua interação com o chatbot ED. O questionário de pós-teste foi criado considerando alguns critérios básicos usuais em processos avaliativos (Hung et al., 2009). É importante mencionar que a inclusão da avaliação do chatbot nessa atividade colaborou para uma análise mais profunda, por parte dos alunos, sobre as dificuldades que envolvem o processo avaliativo de um chatbot.

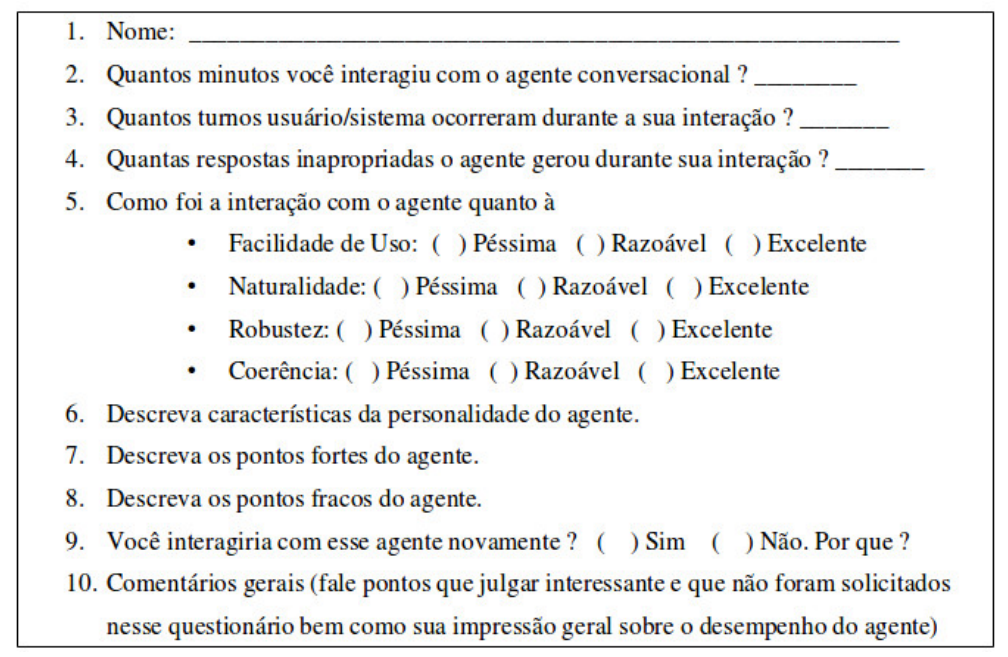

Figura 1. Questionário de pré-teste

Dos 33 alunos da turma, 29 realizaram a tarefa. Sendo que 2 deles não entregaram os questionários. Logo, tivemos cerca de $82 \%$ de efetiva participação. Os respondentes tinham uma média de 25 anos (idade entre 19 e 32 anos). Cerca de $74 \%$ dos alunos nunca tinham interagido com um chatbot antes. Os demais $26 \%$ relataram que já haviam conversado com os chatbots Siri, Eliza, ED, Cleverbot e chatbots em jogos ou de lojas da web. Nenhum dos alunos tinha experiência na prototipação de chatbots, sendo que aproximadamente $93 \%$ indicaram ter conhecimento básico em agentes reativos e apenas 7\%, conhecimento intermediário. Sendo esses últimos, em sua

2 http://www.ed.conpet.gov.br/br/converse.php 
V Congresso Brasileiro de Informática na Educação (CBIE 2016)

Anais do XXII Workshop de Informática na Escola (WIE 2016)

maioria, alunos que já haviam cursado (parcialmente) a disciplina. Quanto ao conhecimento em processamento de linguagem natural (PLN), 85\% indicou conhecimento básico e 15\%, intermediário. Quanto a conhecimentos em IA, 93\% relataram conhecimento básico e os demais $7 \%$, conhecimento intermediário. Igualmente, esses alunos que indicaram conhecimento intermediário também haviam cursando a disciplina anteriormente.

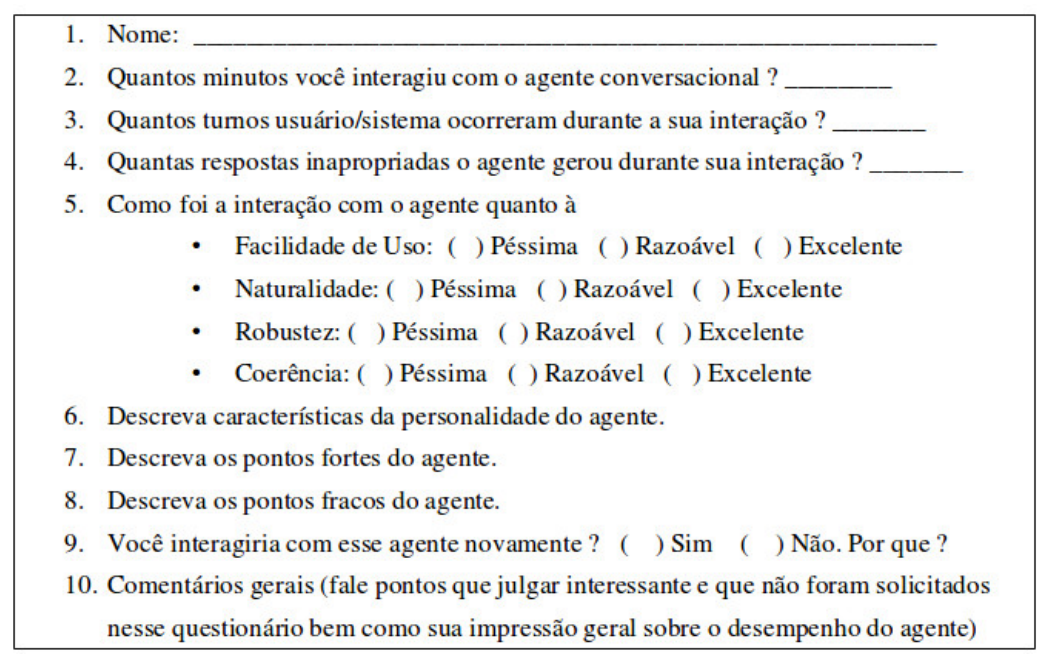

Figura 2. Questionário de pós-teste

Com base nas respostas, observamos que os alunos interagiram, em média, 16 minutos com o chatbot ED. Relataram que, ao longo da conversa, o agente gerou uma média de 11 respostas inapropriadas em cada interação. Apesar de acharem o chatbot razoável (Tabela 1), 96\% dos alunos disse que interagiria novamente com o agente. A maioria dos alunos se surpreendeu positivamente com o desempenho do ED. Além disso, somando-se os conceitos Razoável e Excelente, a maioria dos critérios ficou próximo a $90 \%$. Apenas o critério Coerência ficou um pouco abaixo de $80 \%$ de aceitação. Sob a perspectiva do professor, percebemos que a atividade proposta foi importante, pois a maioria dos alunos nunca havia conversado com um chatbot antes. $\mathrm{O}$ fato dos chatbots serem uma "novidade" foi positivo, pois despertou a curiosidade dos alunos no que se refere à sua construção. Após familiarizar os alunos com a tecnologia, seguimos para a etapa 2 do método.

Tabela 1. Análise de desempenho do chatbot ED.

\begin{tabular}{|l|c|c|c|}
\hline \multicolumn{1}{|c|}{ Critério } & Péssimo & Razoável & Excelente \\
\hline Facilidade de Uso & $0 \%$ & $81 \%$ & $19 \%$ \\
\hline Naturalidade & $0 \%$ & $89 \%$ & $11 \%$ \\
\hline Robustez & $15 \%$ & $74 \%$ & $11 \%$ \\
\hline Coerência & $26 \%$ & $70 \%$ & $4 \%$ \\
\hline
\end{tabular}

\subsection{Construindo um chatbot}

Nessa etapa, a turma foi organizada em equipes. Os alunos se organizaram em 8 equipes com 4 integrantes, em média. Cada equipe definiu os papéis de seus integrantes. A meta das equipes era construir uma parte da base do chatbot. Como o chatbot consista em um agente conversacional reativo, baseamos a implementação da sua base em AIML. 
V Congresso Brasileiro de Informática na Educação (CBIE 2016)

Anais do XXII Workshop de Informática na Escola (WIE 2016)

Escolhemos AIML por ser adequada para agentes reativos, por ser uma linguagem compatível com XML (já conhecida pelos alunos) e ser de fácil aprendizagem.

Uma base AIML contém basicamente o conjunto de perguntas que o agente conversacional é capaz de responder. Essas perguntas são organizadas em categorias. Cada categoria AIML (Figura 3) permite associar um padrão (pattern), que corresponde à sentença de entrada do usuário, a uma resposta (template). Para aumentar a naturalidade, a linguagem permite também que sejam definidas respostas alternativas para uma mesma entrada (random). A linguagem AIML precisa de um interpretador para executar esse casamento de padrões. Decidimos usar pyAIML ${ }^{3}$ por dois motivos: facilidade de instalação e de codificação em Python, que é uma linguagem adequada para processamento de linguagem natural. Além disso, a linguagem Python facilitaria a inclusão de algum tratamento das sentenças de entrada, caso fosse necessário.

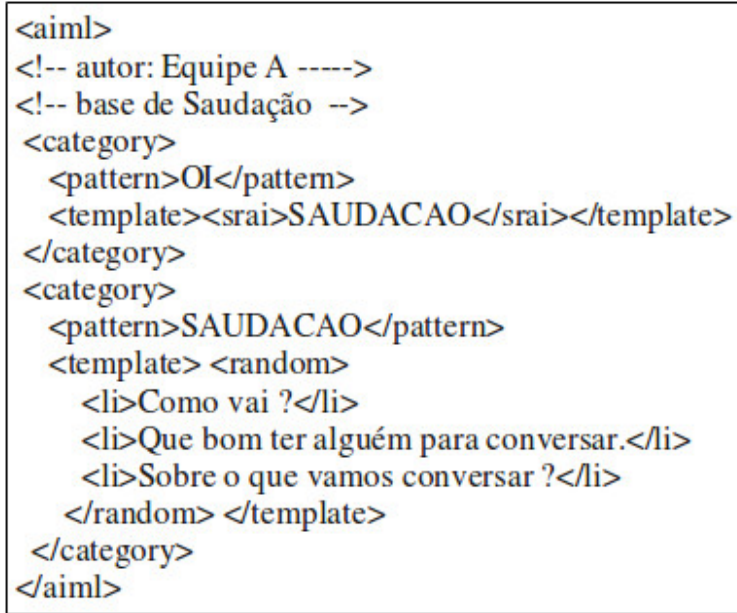

Figura 3. Trecho de uma base AIML

Iniciamos a construção do agente, projetando a sua personalidade e os tópicos sobre os quais o agente seria capaz de conversar. Os alunos se divertiram muito projetando o agente. A Tabela 2 mostra o perfil proposto pelos alunos para o chatbot. Em seguida, atribuídos a cada equipe a responsabilidade de construir uma parte da base de conhecido do agente. Cabe mencionar que os conteúdos de IA a serem incluídos na base do agente foram divididos de forma igualitária às equipes. Apresentamos, na sequência, o básico sobre a linguagem AIML, bem como algumas dicas sobre como estruturar as bases do chatbot. Criamos uma restrição que foi a proibição da incorporação de bases já existentes ao chatbot. O objetivo dessa restrição foi evitar cópias, equiparar o esforço das equipes e estimular o aprendizado a partir da construção da base do agente.

Durante a construção do agente, como esperado, tivemos que incluir alguns tratamentos iniciais às sentenças de entrada. Foi necessário codificar em Python o tratamento da acentuação e de caracteres especiais. Tivemos também que criar um arquivo de substituições para transformar abreviaturas e termos reduzidos usualmente empregados na web (o internetês, exemplo: "vc", "n", “q", ...). Inclusive, esse arquivo de substituições foi um dos problemas críticos durante a fase de integração das bases AIML. Algumas equipes haviam editado o arquivo de substituições e definido formas diferentes de substituição para os mesmos termos.

3 http://sourceforge.net/projects/pyaiml/ 
V Congresso Brasileiro de Informática na Educação (CBIE 2016)

Anais do XXII Workshop de Informática na Escola (WIE 2016)

Essa etapa permitiu ainda que os alunos vivenciassem as dificuldades de integração das bases e os problemas decorrentes dessa integração. Um outro problema que surgiu durante a integração foram os padrões genéricos criados por alguns membros das equipes. Alguns desses padrões tiveram que ser movidos para a base de padrões AIML dita "universais" (que contém os padrões genéricos do agente, criados para gerar respostas para perguntas desconhecidas) para que o chatbot voltasse a responder a perguntas mais específicas corretamente.

Tabela 2. Perfil do chatbot definido pelos alunos.

\begin{tabular}{|c|l|}
\hline Nome & \multicolumn{1}{|c|}{ Ricky } \\
\hline Preferências & $\begin{array}{l}\text { Livro: Guia dos Mochileiros da Galáxia; Série de TV: Game of Thrones; Música: } \\
\text { eletrônica; Esporte: Futebol (gosta, mas não fala muito desde a última copa) e } \\
\text { Xadrez. Time: seleção brasileira; Carro: Google Self-driving Car; Ídolo: Alan } \\
\text { Turing. }\end{array}$ \\
\hline \multirow{2}{*}{ Temas } & $\begin{array}{l}\text { Sistemas de Informação, Área de TI, Universidade, Faculdade, Esportes, } \\
\text { Inteligência Artificial (subáreas: agentes, chatbots e alguns algoritmos de busca } \\
\text { heurística) }\end{array}$ \\
\hline Comportamento & $\begin{array}{l}\text { Bem humorado em geral; empolgado quando fala de suas preferências, exceto no } \\
\text { caso do Futebol; não gosta de ser ofendido mas responde a insultos de forma } \\
\text { educada; é técnico quando fala sobre temas como TI. }\end{array}$ \\
\hline
\end{tabular}

Cabe mencionar que cada equipe, antes da integração, realizou testes em suas bases AIML e que esses testes foram repetidos após a integração. Ao final da prototipação, o agente totalizou 26 bases AIML com aproximadamente 1.450 categorias. Sendo que cada equipe editou em média 180 delas. A maioria dos grupos usou apenas o que foi apresentado em aula quanto aos recursos do AIML. No entanto, as características solicitadas inicialmente foram todas implementadas. A Figura 4 mostra um trecho da base que os alunos implementaram.

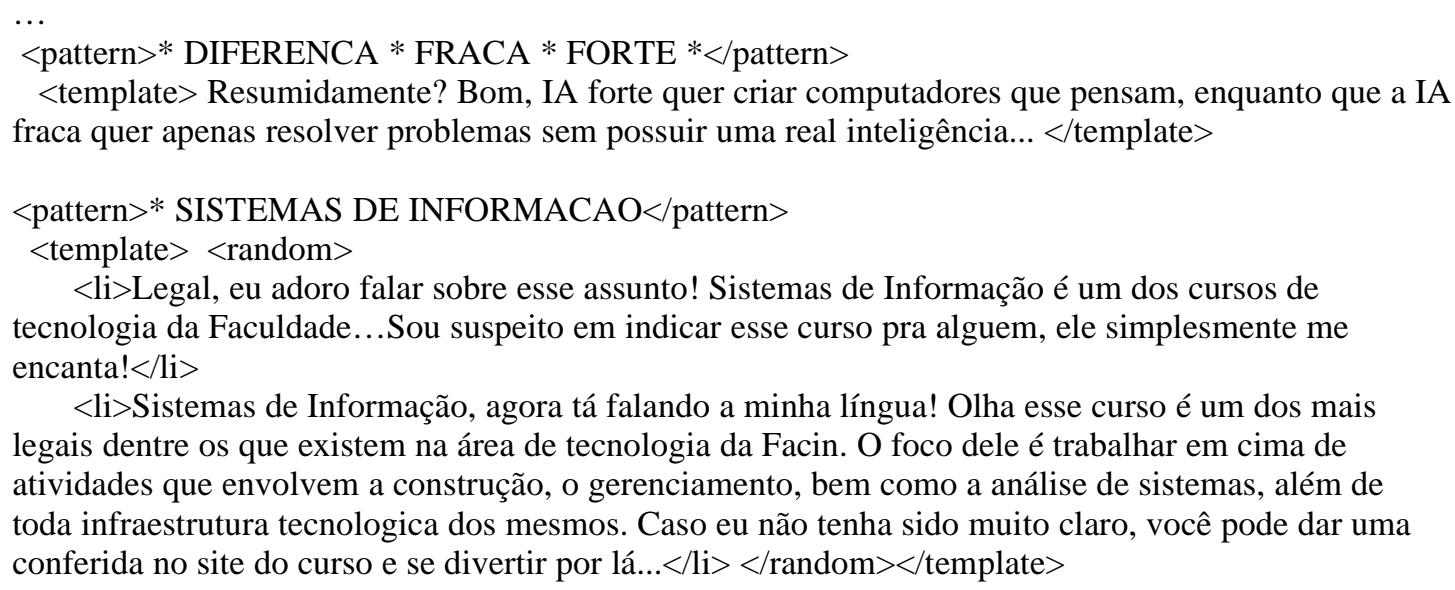

Figura 4.Trecho de uma base em AIML construída pelos alunos (método I)

Construído o agente conversacional, implementamos a fase final.

\subsection{Avaliando um chatbot}

Nessa etapa, solicitamos que os alunos avaliassem o chatbot junto a pessoas que não participaram da sua construção. Para isso, usamos os mesmos questionários de pré e pós-teste apresentados na Seção 4.1. deste artigo. O questionário de pré-teste sofreu algumas adaptações. Foi incluída uma pergunta quanto ao conhecimento em Computação. Em todas as outras áreas foi incluída a opção de resposta "Nenhum" 
(indicando sem conhecimento nas áreas elencadas). No total, 17 pessoas avaliaram o chatbot criado pelos alunos. As pessoas tinham entre 20 e 49 anos e atuavam em diferentes áreas de conhecimento. Apenas duas delas já haviam interagido com um chatbot antes. A maioria dos respondentes não tinha conhecimento algum em agentes reativos, processamento de linguagem natural e inteligência artificial. Quanto ao conhecimento em computação, $44 \%$ tinha conhecimento básico, $50 \%$ conhecimento intermediário ou avançado e apenas $6 \%$ não tinham nenhum conhecimento na área. As pessoas passaram, em média, 9 minutos interagindo com o chatbot Ricky. Comentaram sobre sua personalidade divertida e bem humorada, bem como sua rapidez nas respostas. No entanto, apontaram como pontos fracos a dificuldade do agente em entender as perguntas inseridas pelos usuários e a grande incoerência das respostas. $\mathrm{O}$ chatbot gerava muitas respostas inadequadas. Apesar do baixo desempenho do Ricky, como mostra a Tabela 3, várias pessoas responderam que voltariam a interagir com o agente. Observando-se a Tabela 3, percebemos que para os conceitos Razoável e Excelente, o chatbot Ricky alcançou uma aceitação próxima a 70\%. Para o critério Coerência foi ainda menor, apenas $60 \%$. A maioria dos usuários achou o desempenho do agente apenas Razoável.

Tabela 3. Análise de desempenho do chatbot Ricky.

\begin{tabular}{|l|c|c|c|}
\hline \multicolumn{1}{|c|}{ Critério } & Péssimo & Razoável & Excelente \\
\hline Facilidade de Uso & $29 \%$ & $47 \%$ & $24 \%$ \\
\hline Naturalidade & $29 \%$ & $65 \%$ & $6 \%$ \\
\hline Robustez & $29 \%$ & $65 \%$ & $6 \%$ \\
\hline Coerência & $41 \%$ & $53 \%$ & $6 \%$ \\
\hline
\end{tabular}

Após o processo avaliativo, perguntamos aos alunos o que acharam da experiência e o que tinham aprendido. A Tabela 4 mostra algumas respostas dos alunos. Assim como os alunos, também julgamos a experiência válida. Já esperávamos que chatbot tivesse um baixo desempenho. E isso era previsível por várias razões: as equipes nunca tinham prototipado um chatbot antes; o agente proposto tinha uma arquitetura simples (puramente reativo); e foram incluídos pouquíssimos tratamentos linguísticos. Nosso ganho com esse método, no entanto, não está no agente conversacional em si mas no conhecimento adquirido pelos alunos e nas aptidões que o método proporciona. Com o método, o aluno aprendeu a construir e a avaliar agentes conversacionais reativos. Aprendeu, por meio da construção da base desse agente, mais sobre IA, especialmente sobre agentes reativos. Entendeu, também, as dificuldades que permeiam o tratamento de texto. Além disso, o método incentiva a criatividade, a cooperação e a colaboração. Sem mencionarmos o principal, que foi a participação efetiva da turma na atividade proposta.

Embora a construção de chatbots baseados em AIML envolva ainda muito esforço manual, nem todos os alunos consideraram a atividade maçante. No entanto, percebemos um engajamento maior no início da atividade do que ao seu final. Os alunos notaram de forma concreta os desafios inerentes ao PLN e à construção "por inteiro de um agente conversacional", no tange à definição coerente da personalidade e a naturalidade necessária às interações de um agente conversacional. Uma dificuldade muito comentada por eles foi a integração das bases construídas pelas equipes. O processo de integração e funcionamento coerente do chatbot teve um custo manual além do esperado. Eles perceberam a necessidade de uma maior organização e padronização dos templates usados para definir a base do agente. 
V Congresso Brasileiro de Informática na Educação (CBIE 2016)

Anais do XXII Workshop de Informática na Escola (WIE 2016)

Os comentários dos alunos foram muito positivos e percebemos o ganho do método também nas avaliações realizadas. Na primeira aplicação do método (2015/01), os alunos aumentaram em cerca de $20 \%$ os acertos em questões relacionadas a agentes reativos do que no semestre anterior. O método foi aplicado também no inicio de $2016 \mathrm{e}$ obteve igualmente resultados satisfatórios. Nessa última experiência, também tivemos ganhos significativos na motivação e as notas melhoram nos conteúdos que foram estudados e pesquisados durante a construção da base de conhecimento do agente.

Tabela 4. Avaliação de alguns alunos quanto à atividade proposta

A experiência foi algo novo, já que nunca tínhamos utilizado nenhuma das ferramentas necessárias. No entanto o funcionamento da linguagem aiml mostrou-se simples e sem muitos problemas de implementação.

A experiencia de escrever um chatbot foi muito importante para ajudar a entender os conceitos e limitações que envolvem um agente reativo visto anteriormente na sala de aula.

No desenvolvimento desse trabalho foi possível notar como é complicado e trabalhoso criar um agente reativo para interpretar a Linguagem Natural... Minha visão sobre chattersbots de sites mudou, antes eu não dava bola, agora se visito um site que tem, sempre faço uns testes.

Ao criar chattersbots foi bem massante, contudo deu para ter uma pequena noção de todo o mundo que há de possibilidades para uma simples pergunta.

A experiência de construir o chattersbot foi bem interessante .. ter conhecimento de como é construído um bot foi muito válida.

Foi uma experiência bem interessante, pois já havia interagido antes com outros bots existentes na internet, e é sempre bom saber como as coisas funcionam. Fazendo esse trabalho consegui ter uma melhor compreensão dos mesmos e ví que não é tão simples quanto parece.

Foi uma experiência válida, aprendemos a utilizar o AIML e como funcionam os agentes reativos.

\section{Considerações Finais e Trabalhos Futuros}

Neste artigo apresentamos um método de aprendizagem baseado na prototipação de agentes conversacionais reativos. Julgamos a experiência de aplicação do método satisfatória, pois conseguimos perceber, de forma subjetiva (respostas dos alunos) e objetiva (notas), um ganho na aprendizagem. Percebemos que a atividade de prototipação de chatbot foi motivadora e que, por ser realizada em grupo, estimula, o desenvolvimento de aptidões relacionadas à cooperação e à colaboração. A atividade ainda trabalha a criatividade e a organização dos alunos. Nosso próximo passo é incluir processamento de linguagem natural nos padrões de entrada do usuário para tornar a construção da base AIML mais simples. Pretendemos ainda estudar novas medidas que nos permitam avaliar de forma mais objetiva os efeitos do método na aprendizagem dos alunos.

\section{Agradecimento}

Esta pesquisa tem o apoio financeiro da Pontifícia Universidade Católica do Rio Grande do Sul (EDITAL N. 01/2015 - Chamada para o Programa de Apoio à Atuação de professores Horistas em Atividades de Pesquisa na PUCRS).

\section{Referências}

Al-Zubaide, H. e Issa, A. A. (2011) “OnBot: Ontology based ChatBot". Fourth 
V Congresso Brasileiro de Informática na Educação (CBIE 2016)

Anais do XXII Workshop de Informática na Escola (WIE 2016)

International Symposium on Innovation in Information \& Communication Technology, IEEE, p. 7-12.

Amorim, M. T. C. F; Cury, D. e Menezes, C. S. (2011) "Um sistema inteligente baseado em ontologia para apoio ao esclarecimento de dúvidas ", In: Anais do XXII Simpósio Brasileiro de Informática na Educação - SBIE, 1-9.

Bakar,Ramli. The effect of learning motivation on student's productive competences in vocational high school. International Journal of Asian Social Science, 2014, 4(6), pp. 722-732.

Benotti, L.; Martinez, M. C. e Schapachnik, F. (2014) "Engaging High School Students Using Chatbots, ITICSE, Sweden.

Bii, P. (2013) "Chatbot technology: A possible means of unlocking student potential to learn how to learn". Educational Research, Vol. 4, International Research Journals, 218-221.

Deters, J. I.; Oldoni, A; e Fernandes, A. M. R. (2006) "Bernardo - Agente Pedagógico do Sistema Tutor Inteligente Aplicado a Neurofisiologia". In: Anais do XVII Simpósio Brasileiro de Informática na Educação - SBIE - UNB/UCB.

Domingues, M. J. C. S. e Rothermel, A. (2008) "Chatterbot Application in Business Administration Teaching". 5th CONTECSI - International Conference on Information Systems and Technology Management, 992-1003.

Ferreira, L. P e Uchuoa, J. Q. (2006) "Desenvolvimento de um chatbot para auxiliar o ensino de Espanhol como Língua Estrangeira ". Bazar: Software e Conhecimento Livres, Vol. 1, 21- 32.

Griol, D.; Garcia-Herreno, J. and Molina, J. M. (2011) "The EduAgent Platform: Intelligent Conversational Agents for E-learning Applications". Ambient Intelligence-Sofware and Applications. Advances in Intelligent and Soft Computing, Volume 92, Springer, p. 117-124.

Hung, V.; Elvir, M.; Gonzalez, A.; DeMara, R. (2009), "Towards a method for evaluating naturalness in conversational dialog systems," in Systems, Man and Cybernetics, 2009. SMC 2009. IEEE International Conference on, vol., no., pp.1236-1241.

Jurafsky, D. e Martin, J. H. (2005) Speech and Language Processing: An Introduction to Natural Language Processing, Computational Linguistics, and Speech Recognition.

Kerly, Al.; Ellis, R. and Bull, S. (2009) "Conversational Agents in E-Learning". Applications and Innovations in Intelligent Systems XVI, Springer, p. 169-182.

Moreno, F.C., Manfio, E.R., Barbosa, C.R and Brancher, J.D. (2015) Tical: Chatbot sobre o Atlas Linguístico do Brasil no WhatsApp", CBIE.

Ramayana Junior, A.M and Netto, J. F. M (2014) "Um Chatterbot Educacional Baseado em EmotionML”, CBIE.

Shaw, A. (2012) "Using Chatbots to Teach Socially Intelligent Computing Principles in Introductory Computer Science Courses”, IEEE Computer Society, 850-851.

Tamayo, S. e Pérez-Marín , D. (2012) “An Agent Proposal for Reading Understanding Applied to the Resolution of Maths problems", International Symposium on Computer in Education, IEEE Xplore, 1-4. 